\title{
An Improved D-S Evidence Theory based on Genetic Algorithm to VIP Intelligent Recognition and Recommendation System
}

\author{
Xiaoyin $\mathrm{Xu}^{1}$, Lihong Ren ${ }^{1,2^{*}}$, and Yongsheng Ding ${ }^{1,2^{*}}$ \\ 1) College of Information Sciences and Technology \\ 2) Engineering Research Center of Digitized Textile \& Fashion Technology, Ministry of Education \\ Donghua University, Shanghai 201620, P. R. China \\ * Corresponding to: 1hren@dhu.edu.cn, ysding@dhu.edu.cn
}

\begin{abstract}
In this paper, we use GA to improve the D-S evidence theory, and apply the improved D-S evidence theory to VIP intelligent recognition and recommendation system. In the VIP intelligent recognition and recommendation system of clothes, there are three main evidences: body size, personal preferences, and purchase records. So collision often happens inevitable. This requirement asks us to find out a suitable method to identify the VIPs' needs. D-S evidence theory can improve the rate of identification, but has no idea about the collision. The improved D-S evidence theory based on genetic algorithm can deal with the collision evidence and improve the rate of the identification and the stability. As such we can provide VIP more suitable recommendation. The experiment results of clothes recommendation demonstrate the flexibility of the improved method.
\end{abstract}

Keywords - Information fusion, Genetic algorithm, Evidence theory, Intelligent recommendation.

\section{INTRODUCTION}

Information fusion was initially defined as data fusion. It comes from the Sonar System funded by U.S. Department of Defense in 1973. As a result, the information fusion plays an important role in military field at first. With the passage of time, Information fusion has been studied widely in different fields.

Information fusion is the theory that integrated treating of the multiple sensors or multisource information and making the result more accurate and reliable. In the multisource information system, every piece of information usually is incomplete and inaccurate, even conflicting. Therefore, the essence of information fusion process is to regroup the data and information, estimate and forecast the state of the entity. For now, there are several information fusion algorithms to handle the uncertainty of multisource information, such as Bayesian method, Dempster-Shafer (D-S) evidence theory, fuzzy set, and neural network algorithm etc. As D-S evidence theory has higher recognition rate and reliability with a wide range of applications, it gets more research in practical application.

The development trend in apparel industry is to use hitech application. With the development of Internet of Things (IoT), the hi-tech application is gradually improved. For examples, in an intelligent identification system with RFID for very important person (VIP), the way of recommendation is more and more intelligent. The system uses different irrelevant factors to determine the result of recommendation for customers such as purchase history, favorite color, and body size.

This paper will give a new method about intelligent recognition based on D-S evidence theory improved by genetic algorithm. This method can adapt the intelligent identification for VIP customers and improve the accuracy and the reliability of the identification of VIP customers' information.

The reminder of this paper is organized as follows. The VIP intelligent recognition and recommendation system is depicted in Section II. In Section 3, we propose the improved D-S evidence theory based on genetic algorithm. In Section IV, the experiment results for verification on the clothes recommendation of the proposed method is provided. Section V concludes the whole paper.

\section{ThE VIP INTELLIGENT RECOGNITION AND RECOMMENDATION SYSTEM}

The structure of VIP intelligent recognition and recommendation system is as shown in Fig. 1. It contains VIP intelligent recommendation module, database, and VIP recognition module. The database stores the VIP information and the clothes information both for VIP intelligent recommendation module and VIP recognition module. In VIP recognition module, the system can recognize the VIP's information by RFID tag. In VIP intelligent recommendation module, the system normally requires a combination of body size, personal preferences and purchasing records to give the recommendation to VIP and shows the information on the screen in front of the shop. However, in most cases, these three evidences always conflicts. For example: as show in TABLE I., three evidences give the recommendation about different information. And the clothes' information is shown in TABLE II. In this situation, the system will give the recommendation that is not suitable for VIP. So the system needs a new suitable fusion method to put the three evidences together.

TABLE I. THE AVIDENCES

\begin{tabular}{|c|c|}
\hline Type of evidence & Evidences are given \\
\hline body size & $175 / \mathrm{B}$ \\
\hline personal preferences & Red color, Trousers \\
\hline purchasing records & Red color, Shirt \\
\hline
\end{tabular}


TABLE II. THE CLOTHES ARE AVAILABLE

\begin{tabular}{|c|}
\hline Clothes in the shop \\
\hline Red, $165 / \mathrm{B}$, Trousers \\
\hline Blue, $175 / \mathrm{B}$, Trousers \\
\hline Red, $165 / \mathrm{B}$, Shirt \\
\hline
\end{tabular}

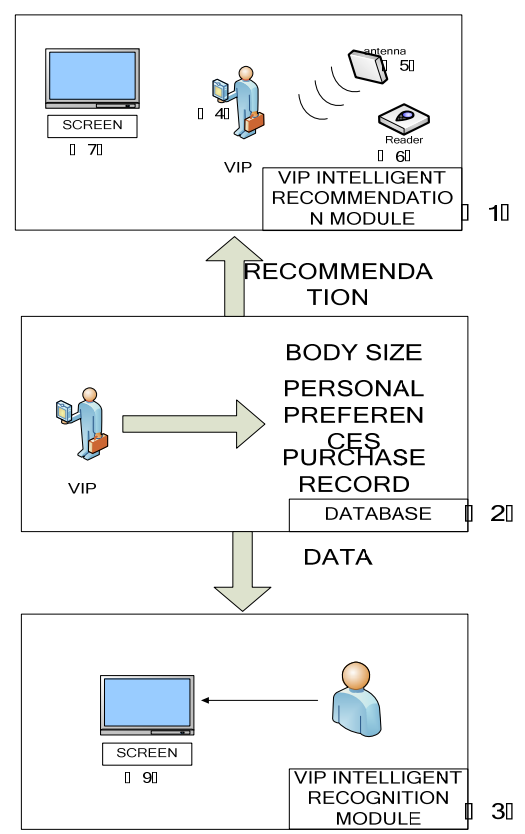

Fig. 1. The structure of VIP intelligent recognition and recommendation system.

\section{THE IMPROVED D-S EVIDENCE THEORY BASED ON GENETIC ALGORITHM}

\section{A. D-S evidence theory}

Define $\Theta$ as a finite non-empty set of hypothesis space, called frame of discernment. Let $2 \mathrm{H}$ be the power set composed of all possible subsets of $\mathrm{H}$. $\mathrm{m}(\mathrm{A})$ is a mapping from $2 \Theta$ to $[0,1]$, and has the following properties:

$$
\begin{aligned}
& m(\phi)=0 \\
& \sum_{A \subseteq \Theta} m(A)=1
\end{aligned}
$$

Then $m(A)$ is called the basic probability assignment (BPA) or belief structure (often referred to as a basic belief assignment, BBA, or a basic mass assignment, BMA).

From the definition of BPA, we can get another two functions about the information fusion. One is denoted as Bel, which is called belief function.

$$
\operatorname{Bel}(A)=\sum_{B \subseteq A} m(B)
$$

Another one is low limited function, which shows the minimum uncertainty of the proposition.

$$
\operatorname{Pl}(A)=1-\operatorname{Bel}(\bar{A})=\sum_{B \cap A \neq \phi} m(B)
$$

The other function is plausibility function, denoted as $\mathrm{Pl}$, which is also a mapping from $2 \Theta$ to $[0,1]$. Plausibility function, that is also called high limited function, shows the sum of all masses of the sets having non-empty intersections.

$\operatorname{Bel}(A)$ and $P l(A)$ are the lower limit and upper limit of the belief level of hypothesis A.

Suppose two independent evidences, $E_{1}$ and $E_{2}$, and their BPA are $m_{1}, m_{2}$. The synthesis evidence $E$ 's $B P A$ is

$$
\begin{aligned}
& m(\phi)=0 \\
& m(C)=\sum_{A \cap B=C} \frac{m_{1}(A) m_{2}(B)}{K}, C \neq \phi \\
& K=1-\sum_{A \cap B=\phi} m_{1}(A) m_{2}(B)=\sum_{A \cap B \neq \phi} m_{1}(A) m_{2}(B)
\end{aligned}
$$

\section{B. Improved D-S evidence theory based on GA}

Genetic Algorithm (GA) is a stochastic optimization search algorithm which simulates the process of biological evolution. GA displays the unique advantages and good performance in many application problems solving combinatorial optimization.

The flowchart of the GA is shown in Fig. 2. The problem is started with an initial solution, which is called population. Every part in population is an answer of the problem, the chromosome. The chromosome in subsequent iteration continuously crossover, mutation and choice, so as to constantly evolution. After several generations, GA converges to the best chromosome. It mostly might be the optimum solution or suboptimal of problem.

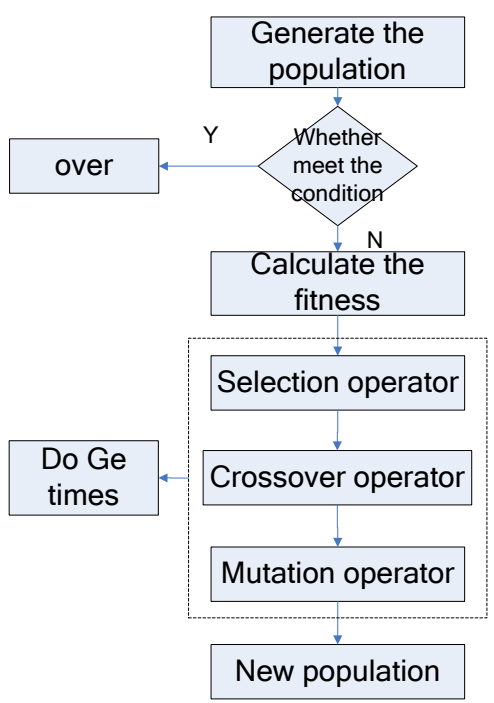

Fig. 2. The flowchart of the GA 
For the GA based D-S evidence theory, there are some new parameters in the new method.

1) Weighted coefficient of evidence conflict:

Define $m_{1}, m_{2}$ as two basic BPA, $x_{1}, x_{2}$ are the weighted coefficient of $m_{1}, m_{2}$. So the weighted coefficient of evidence conflict $K$ can be obtained as:

$$
K=\sum_{A_{i} \cap B_{j}=\Phi} x_{1} m_{1}\left(A_{i}\right) x_{2} m_{2}\left(B_{j}\right)
$$

where $x_{1}+x_{2}=1, x_{1} \in[0,1], x_{2} \in[0,1]$.

2) Weighted evidence distance:

The weighted evidence distance $d$ can be expressed as:

$$
d_{B P A}\left(m_{1}, m_{2}\right)=\sqrt{\frac{1}{2}\left(x_{1} m_{1}-x_{2} m_{2}\right)^{T} D\left(x_{1} m_{1}-x_{2} m_{2}\right)}
$$

where $D(A, B)=\frac{|A \cap B|}{|A \cup B|}, A, B \in P(\Theta)$.

3) Global conflict parameter:

The global conflict parameter is the result of multiinformation cooperation. Suppose the number of evidences is $n$. The number $i$ evidence's weighting is $x_{i}$. We use Eqs. (7) and (8) to update the result

$$
K_{i j m}=0.1 K_{i j}+0.9 d_{i j}
$$

Then we get the global conflict parameter

$$
f=\sum K_{i j m}
$$

\section{The improved D-S evidence theory}

With the three new parameters, the flowchart of the new method is as shown in Fig. 3.

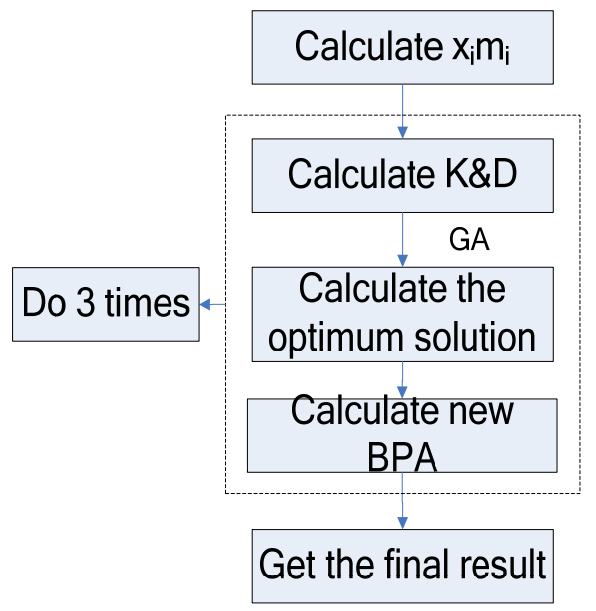

Fig. 3. The improved D-S evidence theory

1) Every evidence multiplies weighting $x_{i}$ to get $x_{i} m_{i}$ and $\mathrm{x}_{\mathrm{i}}$ meets the equation:

$$
x_{1}+x_{2}+x_{3}=1
$$

$$
x_{1}, x_{2}, x_{3} \in[0,1]
$$

2) Caculate every weighted coefficient of evidence conflict $K$ and weighted evidence distance $D$. Use GA to get evidence weighting while the global conflict parameter is minimum.

3) The optimum solution is used to calculate the new weighted average. And a new set of BPA is updatied.

4) Do steps 1)-3) three times, and get the final result.

\section{EXPERIMENT RESULT}

In VIP intelligent recommendation, there are three reference conditions: body size, personal preferences and purchase records. And their BPAs are $m_{1}, m_{2}, m_{3}$.

$$
\begin{aligned}
& m_{1}: m_{1}(a)=0.7, m_{1}(b)=0.1, m_{1}(c)=0.2 \\
& m_{2}: m_{2}(a)=0.8, m_{2}(b)=0.1, m_{2}(c)=0.1 \\
& m_{3}: m_{3}(a)=0.1, m_{3}(b)=0, m_{3}(c)=0.9
\end{aligned}
$$

From the value, there is a conflict in the BPA. You can see $m_{1}, m_{2}$ are different from $m 3$. If we use normal D-S evidence theory, it cannot make the correct decision. But the improved D-S evidence theory based on GA can solve the problem.

As shown in Fig. 3, after calculating by GA, the weighted values are:

$$
x(1)=0.4121, \quad x(2)=0.3696, \quad x(3)=0.2183
$$

So the weighted average value of $m$ is:

$$
\begin{aligned}
& m_{\text {ave }}(a)=0.6060 \\
& m_{\text {ave }}(b)=0.0782 \\
& m_{\text {ave }}(c)=0.3158
\end{aligned}
$$

We do the information fusion for another 2 times with the same way. And the final BPA is:

$$
\begin{aligned}
& m(a)=0.8744 \\
& m(b)=0.0019 \\
& m(c)=0.1237
\end{aligned}
$$

As the result shows that the improved D-S evidence theory based on GA will get the better result. The BPA of three conditions is more like initial BPA. And the conflict will be recognized well. The system can get better recommendation for VIP. The result is as shown in Fig. 4 and Fig. 5. Fig. 4 shows the recommendation with D-S evidence theory. But these clothes don't suit VIP's demands. Because the VIP prefers the red or white clothes rather than black or grey ones. With the improved D-S evidence theory in Fig. 5, these clothes are more suitable for VIP's demands. The three clothes in Fig. 5 are red or white. And the trousers are more suitable for VIP's size. To conclude, the improved D-S evidence theory can calculate more quickly with the three evidences. And the system will give VIPs more attentive service with the new theory. 


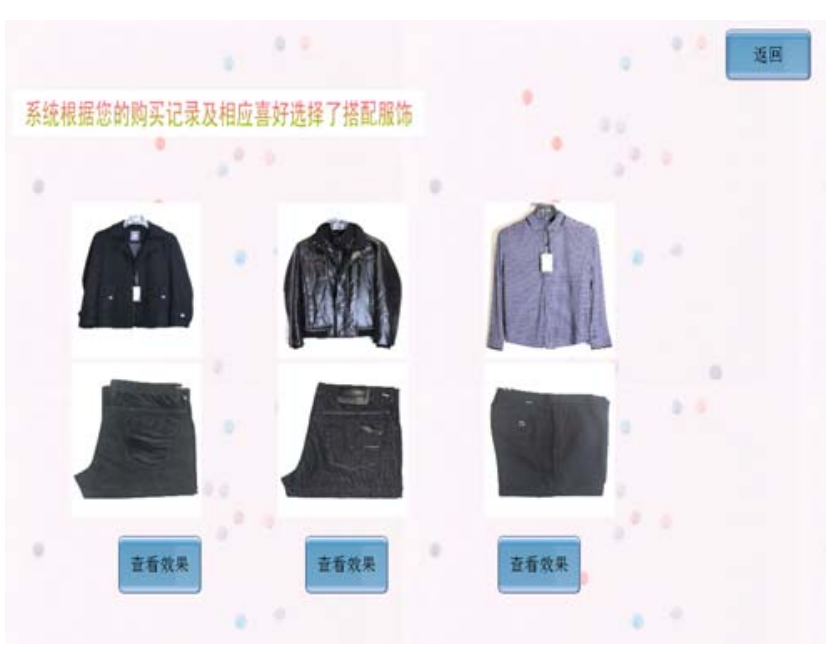

Fig. 4. Recommendation with D-S evidence theory

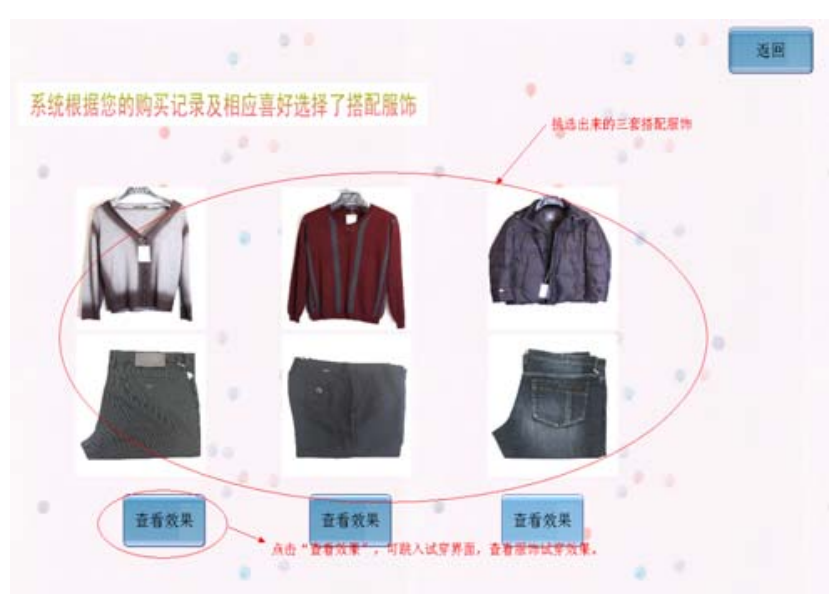

Fig. 5. Recommendation with the improved D-S evidence theory

\section{CONCLUSION}

In this paper, we use GA to improve the D-S evidence theory, and apply the improved D-S evidence theory to VIP intelligent recognition and recommendation system. It contains three evidences: body size, personal preferences, and purchase records. With the new method we can provide VIP more suitable clothes recommendation. It has the better way to solve the conflict situation happened in D-S evidence theory.
Because when the number of evidence becomes larger, the theory should calculate BPA for more times. So the time of the theory gets longer. The next research should calculate the situation about more evidence and how to reduce the calculate time and improve the fusion speed.

\section{ACKNOWLEDGEMENTS}

This work was supported in part by the Key Project of the National Nature Science Foundation of China (No. 61134009), Specialized Research Fund for Shanghai Leading Talents, Project of the Shanghai Committee of Science and Technology (Nos. 11XD1400100, 11JC14002000), and the Fundamental Research Funds for the Central Universities.

\section{REFERENCES}

[1] Baba, Vishwanath V.; HakemZadeh, Farimah, "Toward a theory of evidence based decision making," MANAGEMENT DECISION, vol. 50, pp.832-867, 2012.

[2] Kandara, Osman; Ozturk, Ali, "Kandara, Osman; Ozturk, Ali," ENERGY EDUCATION SCIENCE AND TECHNOLOGY PART A-ENERGY SCIENCE AND RESEARCH, vol. 28, pp.971-982, JAN 2012.

[3] Garcia-Gutierrez, Jorge; Mateos-Garcia, Daniel; Riquelme-Santos, Jose C, "EVOR-STACK: A label-dependent evolutive stacking on remote sensing data fusion " NEUROCOMPUTING, vol. 75, pp. 115122 , JAN 2012.

[4] Khazaee, Meghdad; Ahmadi, Hojat; Omid, Mahmoud, "Vibration condition monitoring of planetary gears based on decision level data fusion using Dempster-Shafer theory of evidence ," JOURNAL OF VIBROENGINEERING, vol. 14, pp. 838-851, JUN 2012.

[5] Banerjee, Tribeni Prasad; Das, Swagatam, "Multi-sensor data fusion using support vector machine for motor fault detection," INFORMATION SCIENCES, vol. 217, Dec. 2012, pp. 96-107.

[6] Bhattacharya, Mahua; Das, Arpita; Chandana, M, "GA-based multiresolution fusion of segmented brain images using PD-, T1-and T2-weighted MR modalities," NEURAL COMPUTING \& APPLICATIONS, vol. 21, SEP. 2012, pp.1433-1447.

[7] Yao, Runming; Yang, Yulan; Li, Baizhan, "A holistic method to assess building energy efficiency combining D-S theory and the evidential reasoning approach," ENERGY POLICY, vol. 45, JUN. 2012, pp. 277-285.

[8] Qi Xue-Mei; Zhang Shao-Cong, “Application of seismic multiattribute fusion method based on D-S evidence theory in prediction of CBM-enriched area ," APPLIED GEOPHYSICS ,vol. 9, MAR. 2012, pp. 80-86.

[9] Sannen, Davy; Van Brussel, Hendrik, "A multilevel information fusion approach for visual quality inspection ," INFORMATION FUSION, vol. 13, JAN. 2012, pp. 48-59.

[10] Matsuzaki, T.; Kameda, H., "Distributed data fusion with data T/R control for target tracking," 2012 Proceedings of SICE Annual Conference, 2012, pp. 701-706. 